\title{
Multiple positive solutions for nonlinear boundary value problem of fractional order differential equation with the Riemann-Liouville derivative
}

\author{
Liu Yang*, Chunfang Shen and Dapeng Xie
}

"Correspondence: yliu722@163.com Department of Mathematics, Hefei Normal University, Hefei, Anhui 230061, China

\begin{abstract}
In this paper, by means of the Avery-Peterson fixed point theorem, we establish the existence result of a multiple positive solution of the boundary value problem for a nonlinear differential equation with Riemann-Liouville fractional order derivative. An example illustrating our main result is given. Our results complement previous work in the area of boundary value problems of nonlinear fractional differential equations (Goodrich in Appl. Math. Lett. 23:1050-1055, 2010).

MSC: 26A33; 34B15
\end{abstract}

Keywords: fractional differential equation; positive solution; fixed point; cone

\section{Introduction}

In this paper, we consider the positive solution for the following boundary value problem of the differential equation involving the Riemann-Liouville fractional order derivative:

$$
\begin{aligned}
& D_{0+}^{\alpha} u(t)+f\left(t, u(t), D_{0+}^{\beta} u(t)\right)=0, \quad t \in(0,1), \\
& u^{(i)}(0)=0, \quad 0 \leq i \leq n-2, \\
& {\left[D_{0+}^{\delta} u(t)\right]_{t=1}=0, \quad 1 \leq \delta \leq n-2,}
\end{aligned}
$$

where $n-1<\alpha \leq n, n>3, n \in \mathbb{N}^{+}, 0<\beta \leq 1$ and $f \in C\left([0,1] \times \mathbb{R}^{+} \times \mathbb{R}, \mathbb{R}^{+}\right)$.

Due to the development of the theory of fractional calculus and its applications, such as in the fields of physics, Bode's analysis of feedback amplifiers, aerodynamics and polymer rheology, etc., many works on the basic theory of fractional calculus and fractional order differential equations have been published [1-7]. Recently, there have been many papers dealing with the solutions or positive solutions of boundary value problems for nonlinear fractional differential equations (FBVPs); see [8-27] and references along this line.

For example, Bai and Lü [12] considered the following Dirichlet boundary value problem of fractional differential equation:

$$
D_{0+}^{\alpha} u(t)+f(t, u(t))=0, \quad t \in(0,1), u(0)=0=u(1), 1<\alpha \leq 2 .
$$

O2014 Yang et al.; licensee Springer. This is an Open Access article distributed under the terms of the Creative Commons Attribution License (http://creativecommons.org/licenses/by/2.0), which permits unrestricted use, distribution, and reproduction in any medium, provided the original work is properly cited. 
By means of some fixed point theorems on cone, existence and multiplicity results of positive solutions were obtained. Furthermore, Bai and Lü proved that the Green's function of problem (1.4) did not satisfy a Harnack-like inequality, which is a crucial property when seeking the existence of positive solutions by means of cone theory. Jiang and Yuan [18] discussed some new positive properties of the Green's function for problem (1.4) and established the existence results of positive solution. The results were established by using the fixed point theorem on a cone due to Krasnoselskii. Recently, Caballero et al. [19] obtained the existence and uniqueness of positive solution for problem (1.4) where the nonlinear term $f$ may be singular at $t=0$. There are also a few papers dealing with the solutions and positive solutions for nonlocal FBVPs; see [22-28].

In a very recent paper, Goodrich [27] considered a class of fractional boundary value problem of the form

$$
\begin{aligned}
& D_{0+}^{\alpha} u(t)+f(t, u(t))=0, \quad t \in(0,1), \\
& u^{(i)}(0)=0, \quad 0 \leq i \leq n-2, \\
& {\left[D_{0+}^{\delta} u(t)\right]_{t=1}=0, \quad 1 \leq \delta \leq n-2,}
\end{aligned}
$$

where $n-1<\alpha \leq n, n \in \mathbb{N}^{+}$and $f \in C\left([0,1] \times \mathbb{R}^{+}, \mathbb{R}^{+}\right)$and $D_{0+}^{\alpha}$ is the standard RiemannLiouville fractional derivative of order $\alpha$. The author obtained the Green's function of this problem and proved that the Green's function satisfied a Harnack-like inequality. By using a fixed point theorem due to Krasnoselskii, the author established the existence of at least one positive solution of problem (1.5)-(1.7).

Problem (1.1)-(1.3) is of interest because it generalizes many problems in real model and have received much attention in recent years. For example, for $\alpha=4$, problem (1.1)-(1.3) may be applied to the description of the deformation of beam and has been studied with a variety of boundary conditions and nonlinearities; see, for example, $[28,29]$ and references therein.

However, in these works, the existence results of positive solutions for FBVPs were all established under the assumption that the derivative of the unknown function $u(t)$ was not involved in the nonlinear term explicitly. To the best of our knowledge, few papers can be found in the literature for a positive solution of FBVPs where the derivative of the unknown function $u(t)$ is involved in the nonlinear term. The main purpose of this paper is to fill this gap.

In [30], Avery and Peterson gave a new triple fixed point theorem, which can be regarded as an extension of the Leggett-Williams fixed point theorem. By using this method, many results concerning the existence of at least three positive solutions of boundary value problems of differential equation with integer order were established; see [31-36]. For example, by using the Avery-Peterson fixed point theorem, Yang et al. [31] established the existence of at least three positive solutions of the second-order multi-point boundary value problem

$$
\begin{aligned}
& u^{\prime \prime}+f\left(t, u(t), u^{\prime}(t)\right)=0, \quad t \in[0,1], \\
& u^{\prime}(0)=\sum_{i=1}^{m-2} \beta_{i} u^{\prime}\left(\xi_{i}\right), \quad u(1)=\sum_{i=1}^{m-2} \alpha_{i} u\left(\xi_{i}\right),
\end{aligned}
$$


where

$$
\begin{array}{ll}
0<\alpha_{i}<1, & 0<\beta_{i}<1, \quad 0<\xi_{i}<1, \quad i=1,2, \ldots, m-2, \\
\sum_{i=1}^{m-2} \alpha_{i}<1, & \sum_{i=1}^{m-2} \beta_{i}<1 .
\end{array}
$$

Though the Avery-Peterson fixed point theorem is a classical tool in seeking the multiple positive solutions of BVPs of nonlinear differential equations of integer order, it cannot be used directly to investigate the existence of positive solutions of BVPs of nonlinear differential equations of fractional order. The main reason is that we cannot derive the concavity or convexity of the function $u(t)$ by the sign of its fractional order derivative. In this paper, by obtaining some new inequalities of the unknown function and defining a special cone, we overcome the difficulties brought about by the lack of the concavity or convexity of the unknown function. By an application of the Avery-Peterson fixed point theorem, the existence of at least three positive solutions of problem (1.1)-(1.3) is established. An example is given to illustrate the main results of this paper.

\section{Preliminaries}

Definition 2.1 The Riemann-Liouville fractional integral of order $\alpha>0$ of a function $u(t)$ is given by

$$
I_{0+}^{\alpha} u(t)=\frac{1}{\Gamma(\alpha)} \int_{0}^{t}(t-s)^{\alpha-1} u(s) d s
$$

provided the right side is point-wise defined on $(0, \infty)$.

Definition 2.2 The Riemann-Liouville fractional derivative of order $\alpha>0$ of a continuous function $u(t)$ is given by

$$
D_{0_{+}}^{\alpha} u(t)=\frac{1}{\Gamma(n-\alpha)}\left(\frac{d}{d t}\right)^{n} \int_{0}^{t} \frac{u(s)}{(t-s)^{\alpha-n+1}} d s,
$$

where $n=[\alpha]+1$, provided that the right side is point-wise defined on $(0, \infty)$.

For example, for $\lambda>-1$, we have

$$
D_{0+}^{\alpha} t^{\lambda}=\frac{\Gamma(1+\lambda)}{\Gamma(1+\lambda-\alpha)} t^{\lambda-\alpha} .
$$

Lemma 2.1 Let $\alpha>0$. The fractional differential equation $D_{0+}^{\alpha} u(t)=0$ has the solution

$$
u(t)=C_{1} t^{\alpha-1}+C_{2} t^{\alpha-2}+\cdots+C_{n} t^{\alpha-n}
$$

for some $C_{i} \in \mathbb{R}, i=1,2, \ldots, n$, where $n$ is the smallest integer greater than or equal to $\alpha$.

Lemma 2.2 Assume that $u(t)$ with a fractional derivative of order $\alpha>0$. Then

$$
I_{0+}^{\alpha} D_{0+}^{\alpha} u(t)=u(t)+C_{1} t^{\alpha-1}+C_{2} t^{\alpha-2}+\cdots+C_{n} t^{\alpha-n}
$$

for some $C_{i} \in \mathbb{R}, i=1,2, \ldots, n$. 
Lemma 2.3 Let $\alpha>0, \beta>0$ and $f \in L_{p}(0,1)(1 \leq p \leq \infty)$, then the following equation:

$$
I^{\alpha} I^{\beta} f(t)=I^{\alpha+\beta} f(t)
$$

holds almost everywhere on $[0,1]$.

Definition 2.3 Let $E$ be a real Banach space over $\mathbb{R}$. A nonempty convex closed set $P \subset E$ is said to be a cone provided that:

(1) $a u \in P$, for all $u \in P, a \geq 0$;

(2) $u,-u \in P$ implies $u=0$.

Definition 2.4 The map $\varphi$ is said to be a nonnegative continuous convex functional on cone a $P$ of a real Banach space $E$ provided that $\varphi: P \rightarrow[0,+\infty)$ is continuous and

$$
\varphi(t x+(1-t) y) \leq t \varphi(x)+(1-t) \varphi(y), \quad x, y \in P, t \in[0,1]
$$

Definition 2.5 The map $\phi$ is said to be a nonnegative continuous concave functional on cone $P$ of a real Banach space $E$ provided that $\phi: P \rightarrow[0,+\infty)$ is continuous and

$$
\phi(t x+(1-t) y) \geq t \phi(x)+(1-t) \phi(y), \quad x, y \in P, t \in[0,1] .
$$

Let $\gamma, \theta$ be nonnegative continuous convex functionals on $P, \varphi$ be a nonnegative continuous concave functional on $P$ and $\psi$ be a nonnegative continuous functional on $P$. Then for positive numbers $a, b, c$, and $d$, we define the following convex sets:

$$
\begin{aligned}
& P(\gamma, d)=\{x \in P \mid \gamma(x)<d\}, \\
& P(\gamma, \varphi, b, d)=\{x \in P \mid b \leq \alpha(x), \gamma(x) \leq d\}, \\
& P(\gamma, \theta, \varphi, b, c, d)=\{x \in P \mid b \leq \varphi(x), \theta(x) \leq c, \gamma(x) \leq d\},
\end{aligned}
$$

and a closed set

$$
R(\gamma, \psi, a, d)=\{x \in P \mid a \leq \psi(x), \gamma(x) \leq d\}
$$

Lemma 2.4 ([30]) Let $P$ be a cone in Banach space E. Let $\gamma, \theta$ be nonnegative continuous convex functionals on $P, \varphi$ be a nonnegative continuous concave functional on $P$, and $\psi$ be a nonnegative continuous functional on P satisfying

$$
\psi(\lambda x) \leq \lambda \psi(x) \text { for } 0 \leq \lambda \leq 1,
$$

such that for some positive numbers $l$ and $d$,

$$
\varphi(x) \leq \psi(x), \quad\|x\| \leq l \gamma(x)
$$

for all $x \in \overline{P(\gamma, d)}$. Suppose $T: \overline{P(\gamma, d)} \rightarrow \overline{P(\gamma, d)}$ is completely continuous and there exist positive numbers $a, b, c$ with $a<b$ such that 
$\left(\mathrm{S}_{1}\right)\{x \in P(\gamma, \theta, \varphi, b, c, d) \mid \varphi(x)>b\} \neq \emptyset$ and $\alpha(T x)>b$ for $x \in P(\gamma, \theta, \varphi, b, c, d)$;

$\left(\mathrm{S}_{2}\right) \varphi(T x)>b$ for $x \in P(\gamma, \varphi, b, d)$ with $\theta(T x)>c$;

$\left(\mathrm{S}_{3}\right) \quad 0 \notin R(\gamma, \psi, a, d)$ and $\psi(T x)<a$ for $x \in R(\gamma, \psi, a, d)$ with $\psi(x)=a$.

Then $T$ has at least three fixed points $x_{1}, x_{2}, x_{3} \in \overline{P(\gamma, d)}$ such that

$$
\begin{aligned}
& \gamma\left(x_{i}\right) \leq d, \quad i=1,2,3 ; \quad b<\varphi\left(x_{1}\right) ; \\
& a<\psi\left(x_{2}\right), \quad \alpha\left(x_{2}\right)<b ; \quad \psi\left(x_{3}\right)<a .
\end{aligned}
$$

\section{Main results}

Lemma 3.1 ([27]) Given $y(t) \in C[0,1]$. Then the following FBVPs:

$$
\begin{aligned}
& D_{0+}^{\alpha} u(t)+y(t)=0, \quad t \in(0,1), \\
& u^{(i)}(0)=0, \quad 0 \leq i \leq n-2, \\
& {\left[D_{0+}^{\delta} u(t)\right]_{t=1}=0, \quad 1 \leq \delta \leq n-2,}
\end{aligned}
$$

are equivalent to the operator equation

$$
u(t)=\int_{0}^{1} G(t, s) y(s) d s
$$

where

$$
G(t, s)= \begin{cases}\frac{t^{\alpha-1}(1-s)^{\alpha-\delta-1}-(t-s)^{\alpha-1}}{\Gamma(\alpha)}, & 0 \leq s \leq t \leq 1, \\ \frac{t^{\alpha-1}(1-s)^{\alpha-\delta-1}}{\Gamma(\alpha)}, & 0 \leq t \leq s \leq 1 .\end{cases}
$$

Lemma 3.2 ([27]) Let $G(t, s)$ be given as in the statement of Lemma 3.1. Then we find that:

(1) $G(t, s)$ is a continuous function on the unit square $[0,1] \times[0,1]$.

(2) $G(t, s) \geq 0$ for each $(t, s) \in[0,1] \times[0,1]$.

(3) $\max _{t \in[0,1]} G(t, s)=G(1, s)$ for each $s \in[0,1]$.

(4) There is a positive constant $\gamma_{0} \in(0,1)$ such that

$$
\min _{t \in\left[\frac{1}{2}, 1\right]} G(t, s) \geq \gamma_{0} G(1, s), \quad 0<s<1,
$$

where

$$
\gamma_{0}:=\min \left\{\frac{1}{\left(2^{\delta}-1\right) 2^{\alpha-\delta-1}}, \frac{1}{2^{\alpha-1}}\right\}>0 .
$$

Lemma 3.3 Given $y(t) \in C[0,1]$. Assume that $u(t)$ is a solution of problem (3.1)-(3.3), then

$$
\left(D_{0+}^{\beta} u\right)(t)=-\frac{1}{\Gamma(\alpha-\beta)} \int_{0}^{t}(t-s)^{\alpha-\beta-1} y(s) d s+\frac{t^{\alpha-\beta-1}}{\Gamma(\alpha-\beta)} \int_{0}^{1}(1-s)^{\alpha-\delta-1} y(s) d s .
$$

Lemma 3.4 Assume that $y(t)>0$ and $u(t)$ is a solution of problem (3.1)-(3.3). Then there exists a positive constant $\gamma_{1}$ such that

$$
\max _{0 \leq t \leq 1}|u(t)| \leq \gamma_{1} \max _{0 \leq t \leq 1} D^{\beta}|u(t)|,
$$

where $\gamma_{1}=\frac{\delta \Gamma(\alpha-\beta)}{(\delta-\beta) \Gamma(\alpha)}>0$. 
Proof From Lemmas 3.1-3.3, we have

$$
\begin{aligned}
& \max _{0 \leq t \leq 1}|u(t)|=\int_{0}^{1} G(1, s) y(s) d s=\int_{0}^{1} \frac{(1-s)^{\alpha-\delta-1}-(1-s)^{\alpha-1}}{\Gamma(\alpha)} y(s) d s, \\
& \max _{0 \leq t \leq 1}\left|D_{0+}^{\beta} u(t)\right| \geq\left|D_{0+}^{\beta} u(1)\right|=\int_{0}^{1} \frac{(1-s)^{\alpha-\delta-1}-(1-s)^{\alpha-\beta-1}}{\Gamma(\alpha-\beta)} y(s) d s .
\end{aligned}
$$

Let

$$
h(s)=\frac{\Gamma(\alpha-\beta)}{\Gamma(\alpha)} \frac{(1-s)^{\alpha-\delta-1}-(1-s)^{\alpha-1}}{(1-s)^{\alpha-\delta-1}-(1-s)^{\alpha-\beta-1}}, \quad 0<s<1 .
$$

We can check that $h(s)$ is positive and strictly decreasing on $(0,1)$. Furthermore,

$$
\lim _{s \rightarrow 0^{+}} h(s)=\frac{\delta \Gamma(\alpha-\beta)}{(\delta-\beta) \Gamma(\alpha)}=\gamma_{1}
$$

Then

$$
h(s) \leq \gamma_{1}, \quad s \in(0,1)
$$

Thus,

$$
\begin{aligned}
\max _{0 \leq t \leq 1}|u(t)| & =\int_{0}^{1} \frac{(1-s)^{\alpha-\delta-1}-(1-s)^{\alpha-1}}{\Gamma(\alpha)} y(s) d s \\
& \leq \int_{0}^{1} \gamma_{1} \frac{(1-s)^{\alpha-\delta-1}-(1-s)^{\alpha-\beta-1}}{\Gamma(\alpha-\beta)} y(s) d s \\
& \leq \gamma_{1} \max _{0 \leq t \leq 1}\left|D_{0+}^{\beta} u(t)\right| .
\end{aligned}
$$

Let the space $X=\left\{u \in C[0,1], D_{0+}^{\beta} u(t) \in C[0,1]\right\}$ be endowed with the norm

$$
\|u\|:=\max \left\{\max _{0 \leq t \leq 1}|u(t)|, \max _{0 \leq t \leq 1}\left|D_{0+}^{\beta} u(t)\right|\right\}
$$

It is well known that $X$ is a Banach space [37]. Define the cone $K \subset X$ by

$$
K=\left\{u \in X: u(t) \geq 0, \min _{t \in\left[\frac{1}{2}, 1\right]} u(t) \geq \gamma_{0} \max _{0 \leq t \leq 1} u(t), \max _{0 \leq t \leq 1}|u(t)| \leq \gamma_{1} \max _{0 \leq t \leq 1} D_{0+}^{\beta}|u(t)|\right\}
$$

Lemma 3.5 Let $T: K \rightarrow X$ be the operator defined by

$$
T u(t):=\int_{0}^{1} G(t, s) f\left(s, u(s), D_{0+}^{\beta} u(s)\right) d s .
$$

Then $T: K \rightarrow K$ is completely continuous.

Proof The operator $T: K \rightarrow X$ is continuous in view of the nonnegativity and continuity of the functions $G(t, s)$ and $f\left(t, u(t), D_{0+}^{\beta} u(t)\right)$. Let $\Omega \subset K$ be bounded. Then there exists a positive constant $R_{1}>0$ such that $\|u\| \leq R_{1}, u \in \Omega$. Denote

$$
R=\sup _{0 \leq t \leq 1, u \in \Omega}\left|f\left(t, u(t), D_{0+}^{\beta} u(t)\right)\right|+1
$$


Then for $u \in \Omega$, we have

$$
\begin{aligned}
|T u| \leq \int_{0}^{1} G(t, s)\left|f\left(s, u(s), D_{0+}^{\beta} u(s)\right)\right| d s \\
\leq R \int_{0}^{1} G(1, s) d s=\frac{\delta R}{\Gamma(\alpha+1)(\alpha-\delta)}, \\
\left|D_{0+}^{\beta}(T u)(t)\right|=\mid-\frac{1}{\Gamma(\alpha-\beta)} \int_{0}^{t}(t-s)^{\alpha-\beta-1} f\left(s, u(s), D_{0+}^{\beta} u(s)\right) d s \\
\quad+\frac{t^{\alpha-\beta-1}}{\Gamma(\alpha-\beta)} \int_{0}^{1}(1-s)^{\alpha-\delta-1} f\left(s, u(s), D_{0+}^{\beta} u(s)\right) d s \mid \\
\leq \frac{R}{\Gamma(\alpha-\beta)}\left(\frac{1}{\alpha-\beta}+\frac{1}{\alpha-\delta}\right) .
\end{aligned}
$$

Hence $T(\Omega)$ is bounded. For $u \in \Omega, t_{1}, t_{2} \in[0,1]$, one has

$$
\begin{aligned}
\left|T u\left(t_{2}\right)-T u\left(t_{1}\right)\right| & \\
\leq & \frac{\left|t_{2}^{\alpha-1}-t_{1}^{\alpha-1}\right|}{\Gamma(\alpha)} \int_{0}^{1}(1-s)^{\alpha-\delta-1} f\left(s, u(s), D_{0+}^{\beta} u(s)\right) d s \\
& +\left|\frac{1}{\Gamma(\alpha)}\left(\int_{0}^{t_{2}}\left(t_{2}-s\right)^{\alpha-1} f\left(s, u, D_{0+}^{\beta} u\right) d s-\int_{0}^{t_{1}}\left(t_{1}-s\right)^{\alpha-1} f\left(s, u, D_{0+}^{\beta} u\right) d s\right)\right| \\
\leq & {\left[\frac{\left|t_{2}^{\alpha-1}-t_{1}^{\alpha-1}\right|}{\Gamma(\alpha)(\alpha-\delta)}+\frac{\left|t_{2}^{\alpha}-t_{1}^{\alpha}\right|}{\Gamma(\alpha+1)}\right] \times R, } \\
\mid D_{0+}^{\beta} & \left(T u\left(t_{2}\right)\right)-D_{0+}^{\beta}\left(T u\left(t_{1}\right)\right) \mid \\
\leq & \left|\frac{1}{\Gamma(\alpha-\beta)}\left(\int_{0}^{t_{1}}\left(t_{1}-s\right)^{\alpha-\beta-1} f\left(s, u, D_{0+}^{\beta} u\right) d s-\int_{0}^{t_{2}}\left(t_{2}-s\right)^{\alpha-\beta-1} f_{1}\left(s, u, D_{0+}^{\beta} u\right) d s\right)\right| \\
& +\left|\frac{t_{2}^{\alpha-\beta-1}-t_{1}^{\alpha-\beta-1}}{\Gamma(\alpha-\beta)} \int_{0}^{1}(1-s)^{\alpha-\delta-1} f\left(s, u(s), D_{0+}^{\beta} u(s)\right) d s\right| \\
\leq & \frac{R}{\Gamma(\alpha-\beta)} \frac{\left|t_{2}^{\alpha-\beta}-t_{1}^{\alpha-\beta}\right|}{\alpha-\beta}+\frac{R}{\Gamma(\alpha-\beta)} \frac{\left|t_{2}^{\alpha-\beta-1}-t_{1}^{\alpha-\beta-1}\right|}{\alpha-\delta} .
\end{aligned}
$$

Thus,

$$
\left\|T u\left(t_{2}\right)-T u\left(t_{1}\right)\right\| \rightarrow 0 \quad \text { for } t_{1} \rightarrow t_{2}, u \in \Omega .
$$

By means of the Arzela-Ascoli theorem, we claim that $T$ is completely continuous. Finally, we see that

$$
\begin{aligned}
\min _{1 / 2 \leq t \leq 1}|T u(t)| & \geq \gamma_{0} \int_{0}^{1} G(1, s) f\left(s, u(s), D_{0+}^{\beta} u(s)\right) d s \\
& =\gamma_{0} \max _{0 \leq t \leq 1} \int_{0}^{1} G(t, s) f\left(s, u(s), D_{0+}^{\beta} u(s)\right) d s \\
& =\gamma_{0} \max _{0 \leq t \leq 1}(T u)(t), \\
\max _{0 \leq t \leq 1}|T u(t)| & =\int_{0}^{1} \frac{(1-s)^{\alpha-\delta-1}-(1-s)^{\alpha-1}}{\Gamma(\alpha)} f\left(s, u(s), D_{0+}^{\beta} u(s)\right) d s
\end{aligned}
$$




$$
\begin{aligned}
& \leq \int_{0}^{1} \gamma_{1} \frac{(1-s)^{\alpha-\delta-1}-(1-s)^{\alpha-\beta-1}}{\Gamma(\alpha-\beta)} f\left(s, u(s), D_{0+}^{\beta} u(s)\right) d s \\
& \leq \gamma_{1} \max _{0 \leq t \leq 1}\left|D_{0+}^{\beta}(T u)(t)\right| .
\end{aligned}
$$

Thus, we show that $T: K \rightarrow K$ is a completely continuous operator.

Let the nonnegative continuous concave functional $\varphi$, the nonnegative continuous convex functionals $\gamma, \theta$ and the nonnegative continuous functional $\psi$ be defined on the cone by

$$
\gamma(u)=\max _{0 \leq t \leq 1}\left|D_{0+}^{\beta} u(t)\right|, \quad \theta(u)=\psi(u)=\max _{0 \leq t \leq 1}|u(t)|, \quad \varphi(u)=\min _{\frac{1}{2} \leq t \leq 1}|u(t)| .
$$

By Lemmas 3.3 and 3.4, the functionals defined above satisfy

$$
\gamma_{0} \theta(u) \leq \varphi(u) \leq \theta(u)=\psi(u), \quad\|u\| \leq \gamma_{2} \gamma(u), \quad u \in P,
$$

where $\gamma_{2}=\max \left\{\gamma_{1}, 1\right\}$. Therefore condition (2.2) of Lemma 2.4 is satisfied.

Assume that there exist constants $0<a, b, d$ with $a<b<d, c=\frac{b}{\gamma_{0}}$ and

$$
\Gamma(\alpha+1)(2 \alpha-\delta-\beta) b<\gamma_{0} \delta \Gamma(\alpha-\beta)(\alpha-\beta) d
$$

such that

$$
\begin{aligned}
& \text { (A } \left.\mathrm{A}_{1}\right) \quad f(t, u, v) \leq \frac{\Gamma(\alpha-\beta)(\alpha-\beta)(\alpha-\delta)}{2 \alpha-\delta-\beta} d, \quad(t, u, v) \in[0,1] \times\left[0, \gamma_{2} d\right] \times[-d, d] ; \\
& \left(\mathrm{A}_{2}\right) \quad f(t, u, v)>\frac{\Gamma(\alpha+1)(\alpha-\delta)}{\gamma_{0} \delta} b, \quad(t, u, v) \in[0,1] \times\left[b, \frac{b}{\gamma_{0}}\right] \times[-d, d] ; \\
& \left(\mathrm{A}_{3}\right) \quad f(t, u, v)<\frac{\Gamma(\alpha+1)(\alpha-\delta)}{\delta} a, \quad(t, u, v) \in[0,1] \times[0, a] \times[-d, d] .
\end{aligned}
$$

Theorem 3.1 Under assumptions $\left(\mathrm{A}_{1}\right)-\left(\mathrm{A}_{3}\right)$, problem (1.1)-(1.3) has at least three positive solutions $u_{1}, u_{2}, u_{3}$ satisfying

$$
\begin{array}{ll}
\max _{0 \leq t \leq 1}\left|D_{0+}^{\beta} u(t)\right| \leq d, & i=1,2,3 ; \\
b<\min _{1 / 2 \leq t \leq 1}\left|u_{1}(t)\right|, & a<\max _{0 \leq t \leq 1}\left|u_{2}(t)\right| ; \\
\min _{1 / 2 \leq t \leq 1}\left|u_{2}(t)\right|<b, & \max _{0 \leq t \leq 1}\left|u_{3}(t)\right| \leq a .
\end{array}
$$

Proof Problem (1.1)-(1.3) has a solution $u=u(t)$ if and only if $u$ solves the operator equation

$$
u(t)=\int_{0}^{1} G(t, s) f\left(s, u(s), D_{0+}^{\beta} u(s)\right) d s=(T u)(t) .
$$

For $u \in \overline{P(\gamma, d)}$, we have $\gamma(u)=\max _{0 \leq t \leq 1}\left|D_{0+}^{\beta} u(t)\right|<d$. From assumption $\left(\mathrm{A}_{1}\right)$, we obtain

$$
f\left(t, u(t), D_{0+}^{\beta} u(t)\right) \leq \frac{\Gamma(\alpha-\beta)(\alpha-\beta)(\alpha-\delta)}{2 \alpha-\delta-\beta} d .
$$


Thus,

$$
\begin{aligned}
\gamma(T u)= & \max _{0 \leq t \leq 1} \mid-\frac{1}{\Gamma(\alpha-\beta)} \int_{0}^{t}(t-s)^{\alpha-\beta-1} f\left(s, u(s), D_{0+}^{\beta} u(s)\right) d s \\
& +\frac{t^{\alpha-\beta-1}}{\Gamma(\alpha-\beta)} \int_{0}^{1}(1-s)^{\alpha-\delta-1} f\left(s, u(s), D_{0+}^{\beta} u(s)\right) d s \mid \\
\leq & {\left[\frac{1}{\Gamma(\alpha-\beta)} \frac{1}{\alpha-\beta}+\frac{1}{\Gamma(\alpha-\beta)} \frac{1}{\alpha-\delta}\right] \times \frac{\Gamma(\alpha-\beta)(\alpha-\beta)(\alpha-\delta)}{2 \alpha-\delta-\beta} d } \\
= & d .
\end{aligned}
$$

Hence, $T: \overline{P(\gamma, d)} \rightarrow \overline{P(\gamma, d)}$.

The facts that the constant function $u(t)=\frac{b}{\gamma_{0}} \in P(\gamma, \theta, \varphi, b, c, d)$ and $\varphi\left(\frac{b}{\gamma_{0}}\right)>b$ imply that $\{u \in P(\gamma, \theta, \varphi, b, c, d \mid \varphi(u)>b)\} \neq \emptyset$. For $u \in P(\gamma, \theta, \varphi, b, c, d)$, we have $b \leq u(t) \leq \frac{b}{\gamma_{0}}$ and $\left|D_{0+}^{\beta} u(t)\right|<d$ for $\frac{1}{2} \leq t \leq 1$. From assumption $\left(\mathrm{A}_{2}\right)$, we see

$$
f(t, u, v)>\frac{\Gamma(\alpha+1)(\alpha-\delta)}{\gamma_{0} \delta} b .
$$

Thus,

$$
\begin{aligned}
\varphi(T u) & \geq \gamma_{0} \max _{0 \leq t \leq 1}\left|\int_{0}^{1} G(t, s) f\left(s, u(s), D_{0+}^{\beta} u(s)\right) d s\right| \\
& \geq \int_{0}^{1} \gamma_{0} G(1, s) d s \times \frac{\Gamma(\alpha+1)(\alpha-\delta)}{\gamma_{0} \delta} b=b,
\end{aligned}
$$

which means $\varphi(T u)>b, \forall u \in P\left(\gamma, \theta, \varphi, b, \frac{b}{\gamma_{0}}, d\right)$. These ensure that condition $\left(\mathrm{S}_{1}\right)$ of Lemma 2.4 is satisfied. Secondly, for all $u \in P(\gamma, \varphi, b, d)$ with $\theta(T u)>\frac{b}{\gamma_{0}}$,

$$
\varphi(T u) \geq \gamma_{0} \theta(T u)>\gamma_{0} \times c=\gamma_{0} \times \frac{b}{\gamma_{0}}=b .
$$

Thus, condition $\left(\mathrm{S}_{2}\right)$ of Lemma 2.4 holds. Finally we show that $\left(\mathrm{S}_{3}\right)$ also holds. We see that $\psi(0)=0<a$ and $0 \notin R(\gamma, \psi, a, d)$. Suppose that $u \in R(\gamma, \psi, a, d)$ with $\psi(x)=a$. Then by assumption $\left(\mathrm{A}_{3}\right)$,

$$
\begin{aligned}
\psi(T u) & =\max _{0 \leq t \leq 1}\left|\int_{0}^{1} G(t, s) f\left(s, u(s), D_{0+}^{\beta} u(s)\right) d s\right| \\
& \leq \int_{0}^{1} G(1, s) d s \times \frac{\Gamma(\alpha+1)(\alpha-\delta)}{\delta} a=a .
\end{aligned}
$$

Thus, all conditions of Lemma 2.4 are satisfied. Hence problem (1.1)-(1.2) has at least three positive solutions $u_{1}, u_{2}, u_{3}$ satisfying

$$
\begin{array}{ll}
\max _{0 \leq t \leq 1}\left|D_{0+}^{\beta} u(t)\right| \leq d, & i=1,2,3 ; \\
b<\min _{1 / 2 \leq t \leq 1}\left|u_{1}(t)\right|, & a<\max _{0 \leq t \leq 1}\left|u_{2}(t)\right| ; \\
\min _{1 / 2 \leq t \leq 1}\left|u_{2}(t)\right|<b, & \max _{0 \leq t \leq 1}\left|u_{3}(t)\right| \leq a .
\end{array}
$$




\section{Example}

Consider the nonlinear FBVPs

$$
\begin{aligned}
& D_{0+}^{\alpha} u(t)+f\left(t, u(t), D_{0+}^{\beta} u(t)\right)=0, \quad t \in(0,1), \\
& u(0)=u^{\prime}(0)=u^{\prime \prime}(0)=0, \quad\left[D_{0+}^{\delta} u(t)\right]_{t=1}=0,
\end{aligned}
$$

where $\alpha=3.6, \delta=1.5, \beta=0.7, n=4$, and

$$
f(t, u, v)= \begin{cases}\frac{1}{\pi^{4}} e^{t}+5 u^{4}+\frac{1}{100} \sin \left(\frac{v}{10,000}\right), & 0 \leq u \leq 7 \\ \frac{1}{\pi^{4}} e^{t}+12,005+\frac{1}{100} \sin \left(\frac{v}{10,000}\right), & u>7\end{cases}
$$

Choose $a=1, b=3, d=10,000$. By a simple computation, we obtain

$$
\begin{aligned}
& \gamma_{0}=\left(\frac{1}{2}\right)^{2.6}, \quad \gamma_{1}=\frac{1.5 \Gamma(2.9)}{0.8 \Gamma(3.6)} \approx 0.9218, \quad \gamma_{2}=1, \\
& \frac{\Gamma(\alpha+1)(\alpha-\delta)}{\delta} a \approx 18.7338, \\
& \frac{\Gamma(\alpha+1)(\alpha-\delta)}{\gamma_{0} \delta} b \approx 340.7416, \\
& \frac{\Gamma(\alpha-\beta)(\alpha-\beta)(\alpha-\delta)}{2 \alpha-\delta-\beta} d \approx 22,257 .
\end{aligned}
$$

We can check that the nonlinear term $f(t, u, v)$ satisfies

(1) $f(t, u, v)<\frac{\Gamma(\alpha-\beta)(\alpha-\beta)(\alpha-\delta)}{2 \alpha-\delta-\beta} d$,

$$
(t, u, v) \in[0,1] \times[0,10,000] \times[-10,000,10,000] ;
$$

(2) $f(t, u, v)>\frac{\Gamma(\alpha+1)(\alpha-\delta)}{\gamma_{0} \delta} b$,

$$
(t, u, v) \in\left[\frac{1}{2}, 1\right] \times\left[3,3 \times 2^{2.6}\right] \times[-10,000,10,000]
$$

(3) $f(t, u, v)<\frac{\Gamma(\alpha+1)(\alpha-\delta)}{\delta} a, \quad(t, u, v) \in[0,1] \times[0,1] \times[-10,000,10,000]$.

Then all assumptions of Theorem 3.1 are satisfied. Thus problem (4.1)-(4.2) has at least three positive solutions $u_{1}(t), u_{2}(t), u_{3}(t)$ satisfying

$$
\begin{aligned}
& \max _{0 \leq t \leq 1}\left|D_{0+}^{\beta} u(t)\right| \leq 10,000, \quad i=1,2,3 ; \\
& 3<\min _{1 / 2 \leq t \leq 1}\left|u_{1}(t)\right|, \quad 1<\max _{0 \leq t \leq 1}\left|u_{2}(t)\right| ; \\
& \min _{1 / 2 \leq t \leq 1}\left|u_{2}(t)\right|<3, \quad \max _{0 \leq t \leq 1}\left|u_{3}(t)\right| \leq 1 .
\end{aligned}
$$

Remark We see that the fractional derivative of function $u(t)$ of order $\beta$ is involved in the nonlinear term of problem (4.1)-(4.2) explicitly. The early results for positive solutions of FBVPs, to the best of our knowledge, are not applicable to this problem. Our results complement some previous works in the area of FBVPs, such as those due to Goodrich [27]. 


\section{Competing interests}

The authors declare that they have no competing interests.

\section{Authors' contributions}

The authors declare that the work was realized in collaboration with the same responsibility. All authors read and approved the final manuscript.

\section{Acknowledgements}

The work is sponsored by the NSFC (11201109), Anhui Provincial Natural Science Foundation (1408085QA07) and the Higher School Natural Science Project of Anhui Province (KJ2012B144, KJ2012Z335).

\section{Received: 8 August 2014 Accepted: 24 September 2014 Published: 03 Nov 2014}

\section{References}

1. Delbosco, D: Fractional calculus and function spaces. J. Fract. Calc. 6, 45-53 (1994)

2. Miller, KS, Ross, B: An Introduction to the Fractional Calculus and Fractional Differential Equations. Wiley, New York (1993)

3. Lakshmikantham, V, Leela, S: Theory of fractional differential inequalities and applications. Commun. Appl. Anal. 11, 395-402 (2007)

4. Lakshmikantham, V, Devi, J: Theory of fractional differential equations in a Banach space. Eur. J. Pure Appl. Math. 1, 38-45 (2008)

5. Lakshmikantham, V, Leela, S: Nagumo-type uniqueness result for fractional differential equations. Nonlinear Anal. TMA 71, 2886-2889 (2009)

6. Lakshmikantham, $\mathrm{V}$, Leela, S: A Krasnoselskii-Krein-type uniqueness result for fractional differential equations. Nonlinear Anal. TMA 71, 3421-3424 (2009)

7. Lakshmikantham, V: Theory of fractional differential equations. Nonlinear Anal. TMA 69, 3337-3343 (2008)

8. Yu, C, Gao, G: Existence of fractional differential equations. J. Math. Anal. Appl. 310, 26-29 (2005)

9. Zhang, S: The existence of a positive solution for a nonlinear fractional differential equation. J. Math. Anal. Appl. 252, 804-812 (2000)

10. Zhang, S: Positive solutions to singular boundary value problem for nonlinear fractional differential equation. Comput. Math. Appl. 59, 1300-1309 (2010)

11. Babakhani, A, Gejji, VD: Existence and positive solutions of nonlinear fractional differential equations. J. Math. Anal. Appl. 278, 434-442 (2003)

12. Bai, Z, Lü, H: Positive solutions for boundary value problem of nonlinear fractional differential equation. J. Math. Anal. Appl. 311, 495-505 (2005)

13. Zhang, S: Positive solutions for boundary-value problems of nonlinear fractional differential equations. Electron. J. Differ. Equ. 2006, 36 (2006)

14. Xu, XJ, Jiang, DQ, Yuan, CJ: Multiple positive solutions for the boundary value problem of a nonlinear fractional differential equations. Nonlinear Anal. TMA 71, 4676-4688 (2009)

15. Su, X: Boundary value problem for a coupled system of nonlinear fractional differential equations. Appl. Math. Lett. 22, 64-69 (2009)

16. Liang, S, Zhang, J: Positive solutions for boundary value problems of nonlinear fractional differential equation Nonlinear Anal. TMA 71, 5545-5550 (2009)

17. Agarwal, RP, O'Regan, D, Staněk, S: Positive solutions for Dirichlet problems of singular nonlinear fractional differential equations. J. Math. Anal. Appl. 371, 57-68 (2010)

18. Jiang, D, Yuan, C: The positive properties of the Green function for Dirichlet-type boundary value problems of nonlinear fractional differential equations and its application. Nonlinear Anal. 15, 710-719 (2010)

19. Caballero, J, Harjani, J, Sadarangani, K: Positive solutions for a class of singular fractional boundary value problems. Comput. Math. Appl. 62, 1325-1332 (2011)

20. Ahmad, B, Nieto, J: Existence results for a coupled system of nonlinear fractional differential equations with three-point boundary conditions. Comput. Math. Appl. 58, 1838-1843 (2009)

21. Bai, Z: On positive solutions of a nonlocal fractional boundary value problem. Nonlinear Anal. TMA 72, 916-924 (2010)

22. Ahmad, B, Sivasundaram, S: On four-point nonlocal boundary value problems of nonlinear integro-differential equations of fractional order. Appl. Math. Comput. 217, 480-487 (2010)

23. Li, C, Luo, X, Zhou, Y: Existence of positive solutions of the boundary value problem for nonlinear fractional differential equations. Comput. Math. Appl. 59, 1363-1375 (2010)

24. Wang, Y, Liu, L, Wu, Y: Positive solutions for a nonlocal fractional differential equation. Nonlinear Anal. TMA 74 3599-3605 (2011)

25. Salem, $\mathrm{H}$ : On the fractional calculus in abstract spaces and their applications to the Dirichlet-type problem of fractional order. Comput. Math. Appl. 59, 1278-1293 (2010)

26. Rehman, $M$, Khan, R: Existence and uniqueness of solutions for multi-point boundary value problems for fractional differential equations. Appl. Math. Lett. 23, 1038-1044 (2010)

27. Goodrich, C: Existence of a positive solution to a class of fractional differential equations. Appl. Math. Lett. 23, 1050-1055 (2010)

28. Graef, J, Yang, B: Positive solutions of a nonlinear fourth order boundary value problem. Commun. Appl. Nonlinear Anal. 14, 61-73 (2007)

29. Ma, R, Xu, L: Existence of positive solutions of a nonlinear fourth order boundary value problem. Appl. Math. Lett. 23, 537-543 (2010)

30. Avery, Rl, Peterson, AC: Three positive fixed points of nonlinear operators on ordered Banach spaces. Comput. Math Appl. 42, 313-322 (2001)

31. Yang, L, Liu, X, Jia, M: Multiplicity results for second-order m-point boundary value problem. J. Math. Anal. Appl. 324 532-542 (2006) 
32. Lian, $\mathrm{H}$, Pang, $\mathrm{H}, \mathrm{Ge}$, W: Triple positive solutions for boundary value problems on infinite intervals. Nonlinear Anal. TMA 67, 2199-2207 (2007)

33. Kosmatov, N: Symmetric solutions of a multi-point boundary value problem. J. Math. Anal. Appl. 309, 25-36 (2005)

34. Pang, H, Lian, H, Ge, W: Multiple positive solutions for second-order four-point boundary value problem. Comput. Math. Appl. 54, 1267-1275 (2007)

35. Shu, X, Xu, Y: Triple positive solutions for a class of boundary value problem of second-order functional differential equations. Nonlinear Anal. TMA 61, 1401-1411 (2005)

36. Feng, $\mathrm{H}, \mathrm{Ge}, \mathrm{W}$ : Existence of three positive solutions for $m$-point boundary-value problems with one-dimensional p-Laplacian. Nonlinear Anal. TMA 68, 2017-2026 (2008)

37. Su, X, Liu, L: Existence of solution for boundary value problem of nonlinear fractional differential equation. Appl. Math. J. Chin. Univ. Ser. B 22, 291-298 (2007)

10.1186/1687-1847-2014-284

Cite this article as: Yang et al:: Multiple positive solutions for nonlinear boundary value problem of fractional

order differential equation with the Riemann-Liouville derivative. Advances in Difference Equations 2014, 2014:284

\section{Submit your manuscript to a SpringerOpen ${ }^{\circ}$ journal and benefit from:}

- Convenient online submission

- Rigorous peer review

- Immediate publication on acceptance

- Open access: articles freely available online

- High visibility within the field

- Retaining the copyright to your article 